\title{
Recurrent desmoid-type fibromatosis associated with underlying neuromuscular choristoma
}

\author{
Jonathan J. Stone, MD, ${ }^{1}$ Nikhil K. Prasad, MBChB, ${ }^{1}$ Pierre Laumonerie, MD, ${ }^{1,2}$ \\ B. Matthew Howe, MD, ${ }^{3}$ Kimberly K. Amrami, MD, ${ }^{3}$ Jodi M. Carter, MD, PhD, ${ }^{4}$ Mark E. Jentoft, MD, ${ }^{4}$ \\ and Robert J. Spinner, MD' \\ 'Department of Neurologic Surgery, Mayo Clinic, Rochester, Minnesota; ${ }^{2}$ Department of Orthopaedic Surgery, Hôpital Pierre \\ Paul Riquet, Toulouse, France; ${ }^{3}$ Department of Radiology, Mayo Clinic, Rochester, Minnesota; and ${ }^{4}$ Department of Laboratory \\ Medicine \& Pathology, Mayo Clinic, Rochester, Minnesota
}

\begin{abstract}
OBJECTIVE Desmoid-type fibromatosis (DTF) presents a therapeutic dilemma. While lacking metastatic potential, it is a locally aggressive tumor with a strong propensity for occurrence near nerve(s) and recurrence following resection. In this study, the authors introduce the association of an occult neuromuscular choristoma (NMC) identified in patients with DTF.

METHODS After experiencing a case of DTF found to have an occult NMC, the authors performed a retrospective database review of all other cases of biopsy-proven DTF involving the extremities or limb girdles in patients with available MRI data. Two musculoskeletal radiologists with expertise in peripheral nerve imaging reviewed the MRI studies of the eligible cases for evidence of previously unrecognized NMC.
\end{abstract}

RESULTS The initial case of a patient with an occult sciatic NMC is described. The database review yielded 40 patients with DTF-18 (45\%) in the upper limb and $22(55 \%)$ in the lower limb. Two cases (5\%) had MRI findings of NMC associated with the DTF, one in the proximal sciatic nerve and the other in the proximal tibial and sural nerves.

CONCLUSIONS The coexistence of NMC may be under-recognized in a subset of patients with extremity DTF. This finding poses implications for DTF treatment and the likelihood of recurrence after resection or biopsy. Further study may reveal crucial links between the pathogenesis of NMC and DTF and offer novel therapeutic strategies.

https://thejns.org/doi/abs/10.3171/2018.3.JNS152935

KEYWORDS choristoma; fibromatosis; aggressive; neoplasm recurrence; local; sciatic nerve; peripheral nerve

$\mathrm{D}$ ESMOID-TYPE fibromatosis (DTF) is a locally aggressive, (myo)fibroblastic neoplasm characterized by an infiltrative growth pattern. Although lacking metastatic potential, it typically widely infiltrates into soft tissues, frequently encompassing neurovascular structures. $^{3-5,12,23-25,27,29-32}$ It can compress, encompass, or invade nerves or track along them, making it difficult to achieve complete excision without compromising nerve function. Recurrence typically happens locally but can occur at distant sites as well, either synchronously or metachronously. The tumor has a multifactorial etiology with genetic, endocrine, and physical features (i.e., trauma) implicated in its pathogenesis. ${ }^{13}$ Mutations in the catenin beta 1 gene (CTNNB1; encoding beta-catenin) are present in approxi- mately $85 \%$ of DTF cases. ${ }^{17}$ While the majority of DTF cases are sporadic, a histologically identical fibromatosis has also been associated with neuromuscular choristoma (NMC). ${ }^{11}$ We have previously reported the presence of CTNNB1 mutations in both NMC and NMC-associated fibromatosis. $^{2}$

Neuromuscular choristoma is a rare peripheral nerve lesion with heterotopic mature skeletal muscle fibers intercalated within nerve fascicles and typically involves major nerve roots or trunks. ${ }^{1}$ Patients with NMC usually present with a progressive neuropathy and associated bony and/or soft-tissue undergrowth. The MRI features include fusiform enlargement of the nerve with minimal enhancement after the intravenous administration of Gd contrast..$^{22}$

ABBREVIATIONS DTF = desmoid-type fibromatosis; LFH = lipofibromatous hamartoma; MRI = magnetic resonance imaging; NMC = neuromuscular choristoma. SUBMITTED December 15, 2015. ACCEPTED March 21, 2018.

INCLUDE WHEN CITING Published online August 31, 2018; DOI: 10.3171/2018.3.JNS152935. 


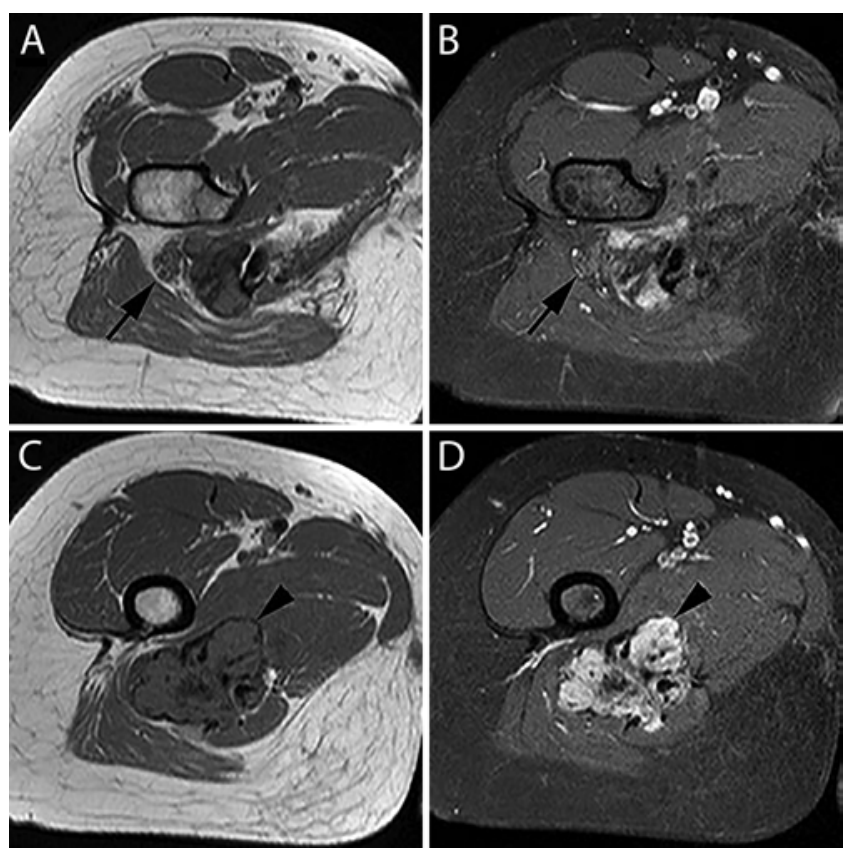

FIG. 1. Case 1. Preoperative MRI. Axial T1-weighted (A) and post-Gd (B) MR images of the proximal right thigh demonstrate an abnormal proximal sciatic nerve (arrows). The nerve is enlarged with prominent soft-tissue elements, signal intensity comparable to skeletal muscle, and no enhancement after contrast, corresponding to the expected features of NMC. Additional axial T1-weighted (C) and post-Gd fat-saturated (D) MR images from the proximal right thigh demonstrate a heterogeneous mass (arrowheads) intimately associated with the proximal sciatic nerve. The mass demonstrates heterogeneous areas of decreased T1- and T2weighted signal intensity and post-Gd enhancement compatible with DTF.

Patients with NMC can develop DTF spontaneously, or following biopsy or resection, in the soft tissues surrounding the biopsy site, in fact, near the NMC. ${ }^{10,11}$ Despite the often striking clinicoradiological features of NMC, these tumors can be overlooked if not actively sought out or not recognized.

In this study, we introduce a subset of patients who presented primarily with DTF and were subsequently found to have an occult underlying NMC. We discuss this new diagnosis, the association of NMC with DTF, and management implications.

\section{Methods}

After encountering the patient in case 1 (described below), we attempted to confirm an association of DTF with occult NMC. Institutional review board approval was obtained before undertaking the database review. We performed a retrospective review of all patients who had been referred to our institution with a pathology-proven diagnosis of DTF between January 2011 and January 2016. We then narrowed our search to include only extra-abdominal cases of DTF such as occurring in the upper and lower limbs, pelvic and pectoral girdles, or retroperitoneal paraspinal regions. Cases without MRI and those without a confirmed pathological diagnosis of DTF were excluded.

Magnetic resonance images were reviewed to confirm the presence of DTF and to identify radiological features of NMC within an adjacent nerve including 1) fusiform enlargement of the nerve, 2) less than $50 \%$ intralesional fat, 3) intraneural soft-tissue components with signal intensity similar to surrounding muscle, and 4) minimal contrast enhancement. All images were reviewed by two musculoskeletal radiologists (B.M.H. and K.K.A.) experienced with NMC and NMC-associated DTF. Pathology results were re-reviewed for DTF and NMC by the same musculoskeletal pathologist (J.M.C.).

\section{Results}

Forty-seven other cases of extremity/limb girdle DTF were identified. Seven cases were excluded because definitive histopathological diagnosis was not available. Of the remaining 40 cases, 18 (45\%) DTFs occurred in the upper limb or pectoral girdle, and $22(55 \%)$ were in the lower limb or pelvic girdle. Two patients (cases 2 and 3) with DTF had MRI features that revealed unrecognized NMC of the associated nerve (5\%). Both patients had recurrent DTF of the lower extremity and had been referred for a second opinion. Case 2 was subsequently evaluated at our institution, and information for case 3 was obtained through chart and imaging review only. Only one of these 2 cases had tissue available for pathological review. The DTF showed the characteristic histological features. No NMC was identified in the available material for review. Lesional DTF tissue was tested for CTNNB1 "hot-spot" mutations, and a $C T N N B 1$ p.S45F mutation was identied.

\section{Illustrative Cases \\ Case 1}

History and Examination

A 34-year-old-woman presented with a 2-year history of primarily right buttock pain that occasionally radiated down her posterior thigh to the dorsal foot. After the pain progressively worsened to the extent that she had trouble sitting in a car and driving, she sought evaluation from multiple medical professionals without a clear diagnosis. Initial examination did not reveal any focal deficits. Electrodiagnostic studies were not done. Magnetic resonance imaging of the lumbar spine did not reveal a structural cause for the pain. A physiotherapist subsequently palpated a mass in the proximal thigh that prompted imaging of the right lower limb. Magnetic resonance imaging (Fig. 1) demonstrated a $5.2 \times 4.1 \times 18-\mathrm{cm}$ (transverse $\times$ anteroposterior $\times$ superoinferior) mass with irregular borders, areas of decreased signal on both T1- and T2-weighted sequences, and heterogeneous post-Gd enhancement. The mass was intimately associated with the sciatic nerve. An image-guided needle core biopsy was performed the following month, and DTF was diagnosed. The patient underwent resection of the mass, with 1-mm lateral, anterior, and posterior margins from the underlying sciatic nerve.

The patient obtained only partial pain relief following the surgery and developed new numbness in her right leg. Follow-up MRI 8 months later demonstrated extensive recurrent fibromatosis involving the sciatic nerve. The pa- 


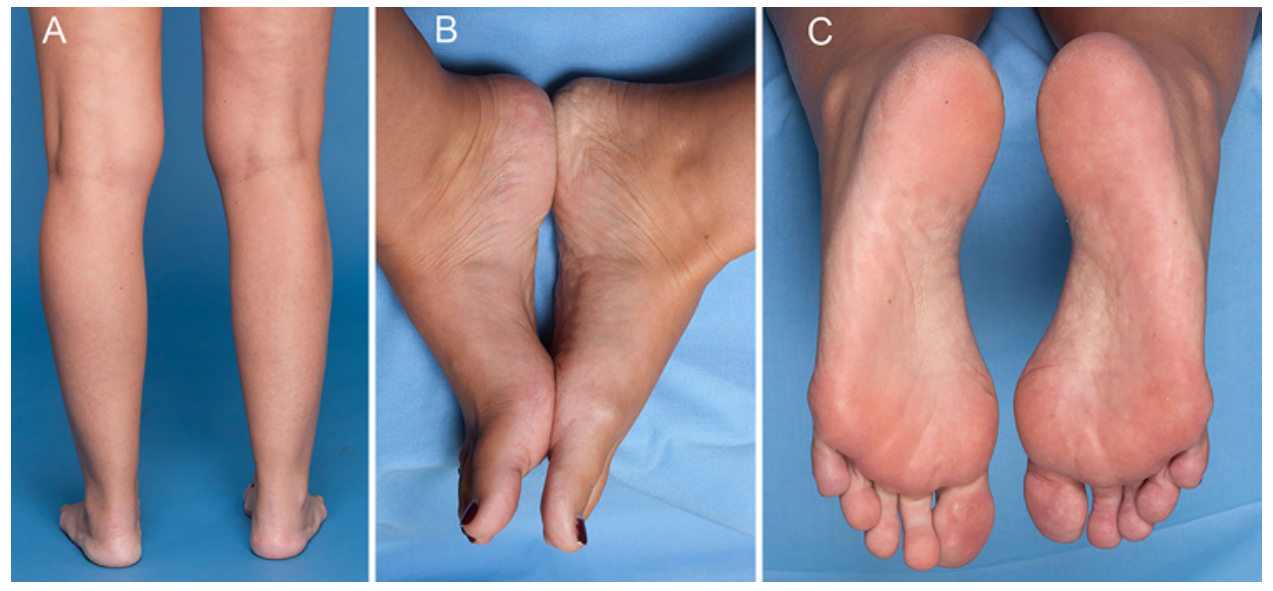

FIG. 2. Case 1. Clinical images demonstrating atrophy of the right calf $(\mathbf{A})$, an asymmetrical right talipes cavus deformity (B), and the right foot shorter than the left (C). The patient was unaware of the high arch but acknowledged the long-standing foot length discrepancy. Figure is available in color online only.

tient was referred to our institution for further evaluation. Interpretation of the tissue specimens by a neuropathologist and a radiologist's review of prior MRI studies agreed with the outside diagnosis of sporadic proximal thigh DTF involving the sciatic nerve.

\section{Reinterpretation of the Imaging}

After the patient's initial episode of care was completed at our institution, two musculoskeletal radiologists (B.M.H. and K.K.A.) experienced with NMC and NMC-associated DTF reviewed the MR images. In addition to mild enlargement of the fibromatosis associated with the sciatic nerve, critical examination revealed two previously unrecognized findings: 1) NMC was present in the sciatic nerve on preoperative imaging (Fig. 1); and 2) there was a linear softtissue abnormality in the popliteal fossa lateral to the semimembranosus muscle that extended to the posterior aspect of the medial gastrocnemius muscle, i.e., a possible separate site of DTF on the short-term follow-up imaging. The nerve exhibited heterogeneous enlargement of individual nerve bundles without significant T2-weighted hyperintensity or post-Gd enhancement and did not have the typical appearance of compressive or reactive neuritis secondary to the fibromatosis. This neural abnormality was consistent with the appearance of an NMC and extended over the entire visualized portion of the sciatic nerve, from the lumbosacral plexus in the sciatic notch to the midthigh.

\section{Reexamination of the Patient}

At this time, the senior author (R.J.S.) reviewed the imaging and predicted that the patient would have clinical features consistent with NMC of the sciatic nerve. On examination, he identified subtle features suggestive of a long-standing and progressive neuropathy with associated atrophy of the calf and undergrowth (including talipes cavus of the right foot and mild shortening of both the right foot and lower limb; Fig. 2). There was mild weakness in plantar flexion and dorsiflexion, a decreased ankle reflex, and preserved sensory function. Scanogram confirmed that the right lower limb was $0.5 \mathrm{~cm}$ shorter than the left.

\section{Pathological Reexamination of the Original Specimen and Molecular Testing}

Given his clinical suspicion of an underlying NMC, the senior author sought pathological confirmation. Careful histopathological re-review of the excised specimen revealed a previously unrecognized microscopic NMC involving a small peripheral nerve branch within the DTF (Fig. 3). Based on our prior observation of the high frequency of CTNNB1 p.S45F mutations in NMC and NMCassociated fibromatosis, the DTF tissue was tested and a CTNNB1 p.S45F mutation was identified. Because of the microscopic amount of NMC lesional tissue, which was embedded within the DTF, it could not be separately tested for $C T N N B 1$ mutations.

\section{Clinical Course}

Approximately 1 year after the initial subtotal resection, the right thigh mass was noted to enlarge, and the patient developed worsening pain requiring narcotic use. A 6-month trial of tamoxifen ( $40 \mathrm{mg}$ daily) was attempted, but there was no significant effect on the symptoms or the tumor volume. The patient subsequently underwent 5940cGy fractionated external beam radiation using a shrinking field technique. This tumor seemed to stabilize on serial imaging; however, the patient noticed a new palpable mass in her popliteal fossa (1 year after completion of the radiation therapy; Fig. 4). A needle biopsy was performed on this second distal lesion, which showed the characteristic histological features of DTF and was also positive for a CTNNB1 p.S45F mutation. Given this second lesion, the patient was placed on a course of Xeloda (capecitabine) but did not tolerate it well, and the drug was stopped after 9 months. She next underwent image-modulated radiotherapy boost of 2000 cGy to the distal lesion because there was overlap from the initial fractionated radiation field. At the time of her most recent follow-up (6 years after clinical presentation, 5 years after the subtotal resection, and 2 years from the development of her second lesion), her symptoms were reasonably well controlled with gabapentin and low-dose (as needed) Percocet. Serial im- 

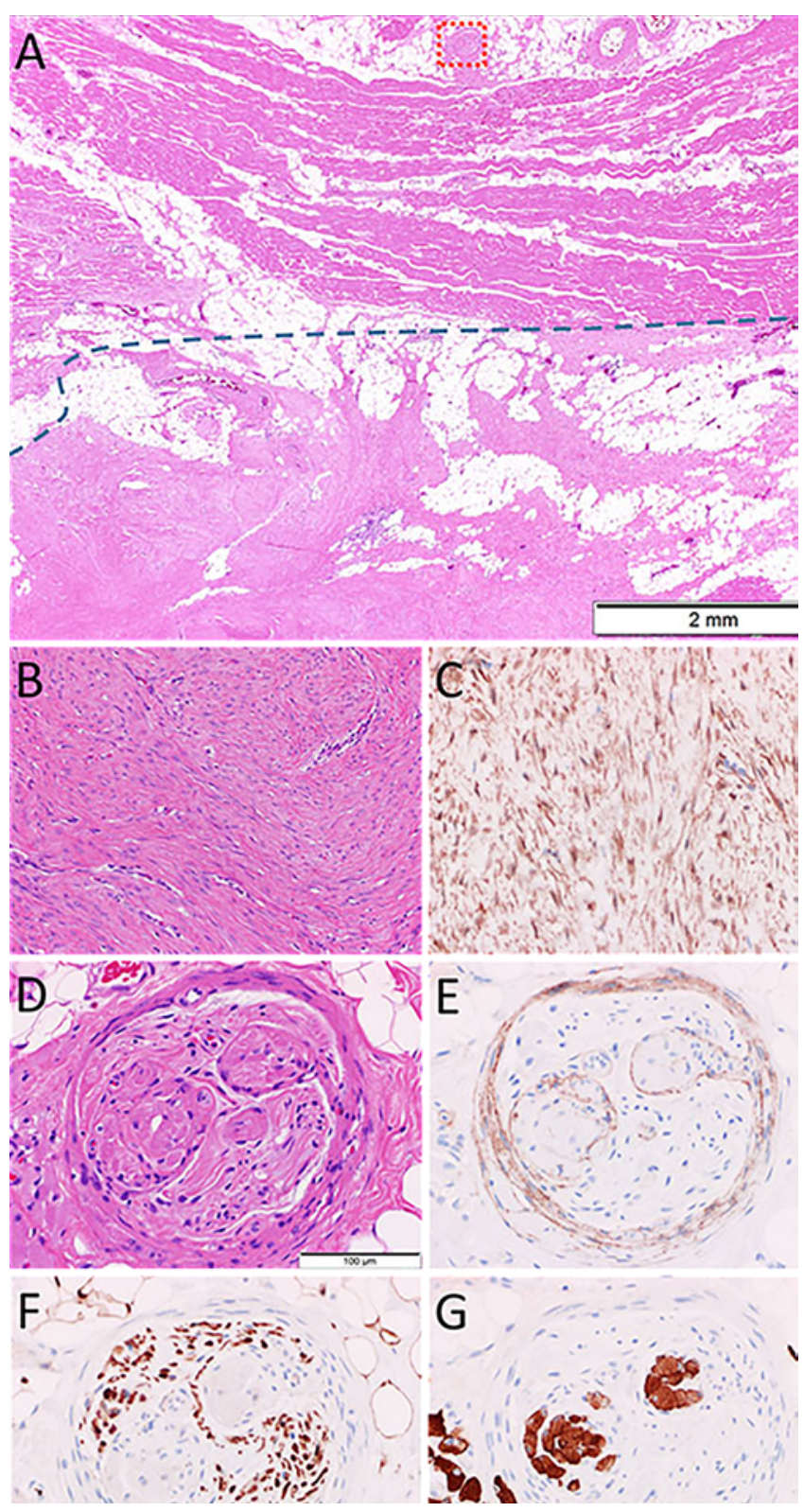

FIG. 3. Case 1. A: The area below the dashed line is DTF infiltrating the soft tissue, whereas the area above the dashed line is composed of mature skeletal muscle and adipose tissue with a small nerve involved by NMC (red dotted box). H \& E, original magnification $\times 20$. B and C: Desmoid-type fibromatosis with a classic histological appearance and containing aberrant nuclear localization of beta-catenin protein. $\mathrm{H}$ \& $E(B)$ and beta-catenin (C), original magnification $\times 200(B)$ and $\times 400(C)$. D-G: Magnification of the red dotted box in panel A. A small nerve (D) found in the soft tissue is within $3 \mathrm{~mm}$ of the DTF. Epithelial membrane antigen (E) highlights perineurium of the small nerve, and $\mathrm{S} 100$ protein (F) highlights the nerve fibers. Desmin $(G)$ highlights the mature skeletal muscle within two of the nerve fascicles as well as skeletal muscle in the adjacent soft tissue. The presence of skeletal muscle intercalated within the nerve fascicles is diagnostic of an NMC. $H$ \& $E(D)$, original magnification $\times 400(D-G)$. Figure is available in color online only. aging showed continuously increasing conspicuity of the NMC within the sciatic nerve, both proximal and distal to the primary mass in the upper thigh, specifically involving both the tibial and peroneal divisions, in continuity with the newly palpable mass in the popliteal fossa.

\section{Case 2}

History and Examination

A 19-year-old man noted a mass in his popliteal fossa, which was sampled with needle biopsy and reported to be a DTF. Magnetic resonance imaging of the popliteal fossa showed a large lobulated mass that encased the popliteal vessels and tibial nerve. There was fusiform enlargement and prominent soft-tissue elements at the most proximal extent of the imaging, consistent with NMC of the tibial division of the sciatic nerve (Fig. 5).

\section{Operation and Postoperative Course}

He underwent en bloc resection including the popliteal vessels and interposition saphenous vein graft. He subsequently developed compartment syndrome, requiring fasciotomy, vascular reconstruction, and ultimate closure with local tissue advancement and skin grafting. Approximately 9 months following surgery, there was a local recurrence and the patient underwent 5000-cGy fractionated external beam radiation. At the time of his last followup (15 months from surgery), there was ongoing growth of the tumor, and the patient was considering a chemotherapeutic approach versus above-knee amputation.

\section{Case 3}

History and Examination

A 20-year-old woman was found to a have a painless mass involving the right gluteus maximus muscle. Magnetic resonance imaging demonstrated a large lesion within the gluteus maximus adjacent to an enlarged sciatic nerve and superior gluteal nerve (Fig. 6).

\section{Operation and Postoperative Course}

She underwent resection of the tumor, reported as a desmoid tumor, followed by fractionated radiation therapy. Three-month postoperative MRI demonstrated gross-total resection of the mass but the persistence of an unrecognized abnormality within the sciatic nerve both proximal and distal to the primary mass. Distally the nerve was abnormal throughout its visualized course to the lower thigh. Unfortunately, she developed a local recurrence (approximately 2 years after surgery) along the course of the abnormal muscular branch, extending toward the greater sciatic notch. She was treated with 4 cycles of Adriamycin and DTIC (dacarbazine), and the tumor seemed to stabilize. During pregnancy, 5 years after her resection, the tumor again began to grow, and the patient noticed a lump in her proximal calf. On MRI, a second lesion consistent with DTF was found in the popliteal fossa (an area not previously well visualized on prior imaging), wrapping around the fibular head, along the course of the lateral sural cutaneous nerve (Fig. 7). This MRI, which included the pelvis to below the knee, showed that the entire course of the sciatic nerve was affected by NMC, especially the pero- 

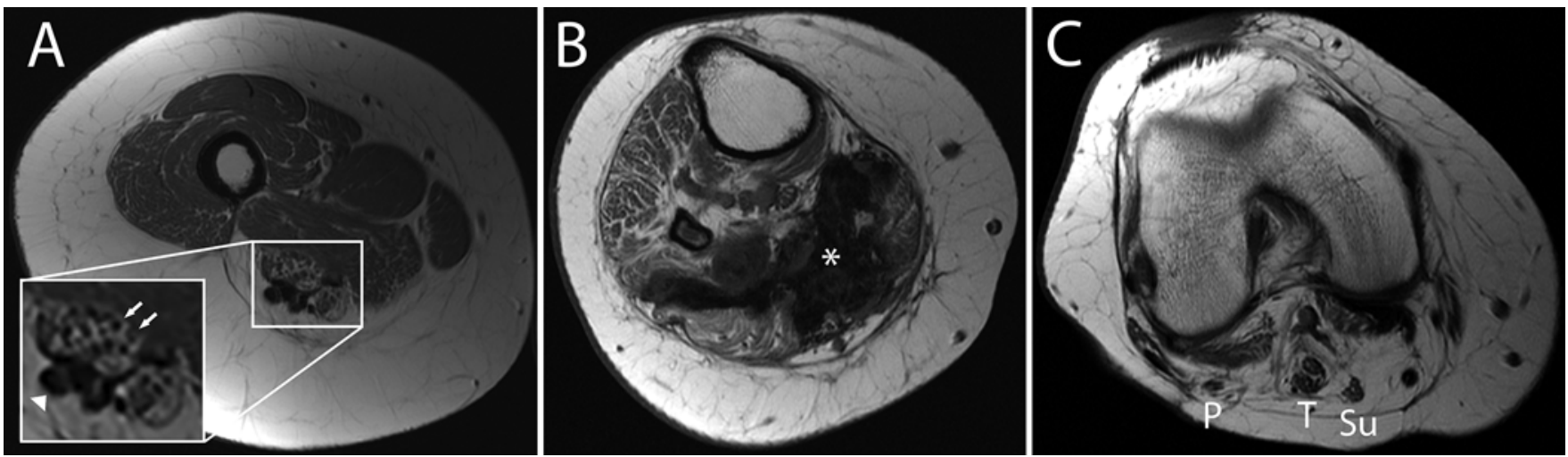

FIG. 4. Case 1. Axial T1-weighted MR image (A) obtained at the inferior aspect of the primary mass in the upper thigh, showing the typical imaging features of NMC in the proximal sciatic nerve with nerve fascicles of varying size (arrows), some of which include soft-tissue elements with signal comparable to that for skeletal muscle. The adjacent low-signal nodular area (arrowhead) is the associated desmoid. Axial T1-weighted MR image (B) obtained distal to the popliteal fossa in the proximal calf, showing the development of an additional focus of fibromatosis (asterisk) with characteristic low signal and ill definition with infiltration of the posterior compartment musculature. Axial T1-weighted MR image (C) obtained in the distal thigh below the sciatic bifurcation but proximal to the desmoid in the calf. There are fat and soft-tissue elements associated with the tibial (T), sural (Su), and peroneal (P) nerves, indicating the presence of NMC within these nerves, in continuity with the more proximal and distal nerves.

neal nerve distal to the bifurcation. Biopsy of this second lesion was consistent with DTF. After delivery, there was continued lesion growth, and she was placed on raloxifene and sulindac. After several months of this therapy, at the time of her last follow-up (6 years from diagnosis), there was continued progression of the popliteal lesion, and the patient was considering cryotherapy.

\section{Discussion}

We identified three patients who had the novel presen- tation of a lower-extremity DTF leading to the diagnosis of an unrecognized NMC. Given our previous reports of the etiological association between these lesions, ${ }^{10,11,22}$ we believe that NMC in this setting is an under-recognized entity and may predispose certain patients to develop multifocal DTF. The radiological features of NMC can be easily overlooked, as in the cases featured here, or misinterpreted, as in lipofibromatous hamartoma (LFH; i.e., lipomatosis of the nerve). ${ }^{18,21}$ While both NMC and LFH present with fusiform enlargement of the proximal nerve trunks, NMC contains $<50 \%$ intralesional fat ${ }^{16}$ and $\mathrm{LFH}$
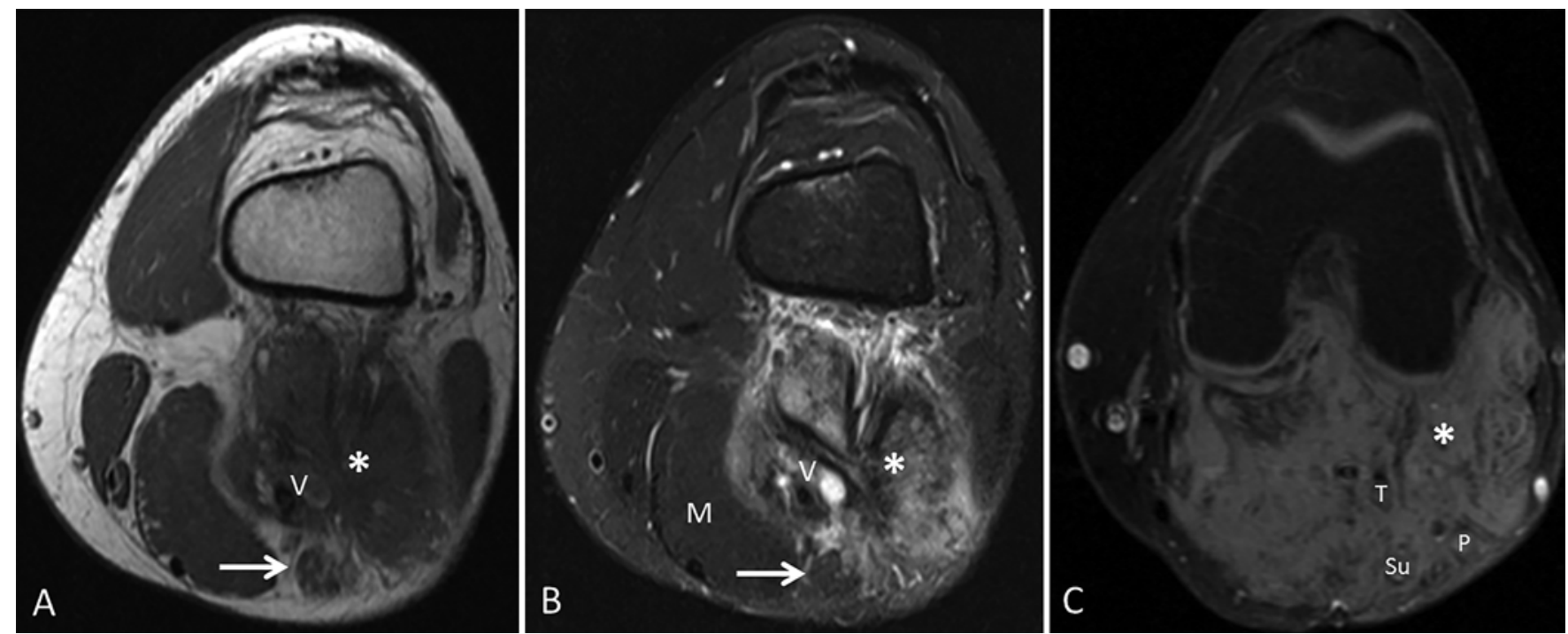

FIG. 5. Case 2. A: Preoperative axial T1-weighted MR image obtained at the upper popliteal fossa, showing fusiform enlargement of the tibial nerve with prominent soft-tissue elements (arrow) compatible with the appearance of an NMC. The nerve is posterior to a lobulated mass (asterisk) consistent with DTF, which encases the popliteal vessels (V). B: Axial T2-weighted MR image obtained at the same level; the tibial nerve (arrow) is isointense with adjacent muscle (M), characteristic of an NMC. C: Postoperative axial T1-weighted MR image with fat suppression at the level of the knee, showing encasement of the enlarged tibial (T), sural (Su), and common peroneal (P) nerves by recurrent DTF (asterisk). 

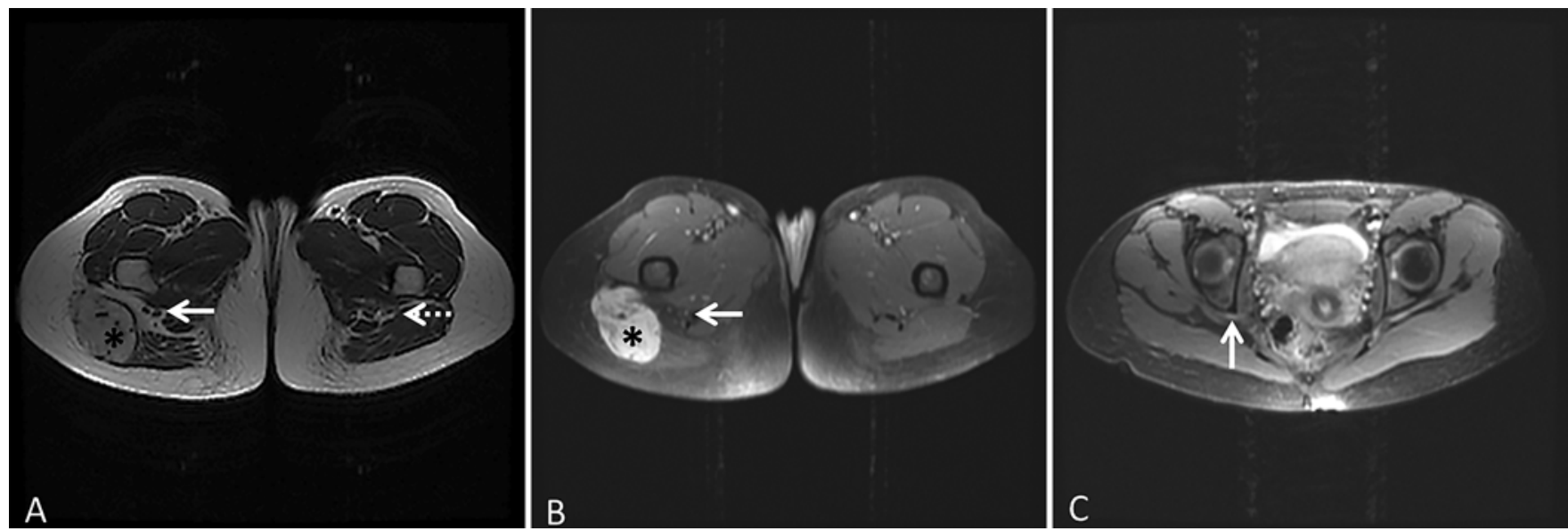

FIG. 6. Case 3. Preoperative axial T1-weighted MR image (A) showing right-sided inferior gluteal DTF (asterisk) posterolateral to the enlarged sciatic nerve (solid arrow). The nerve on the right side is noticeably larger than the normal left (dashed arrow). After contrast administration (B), the sciatic nerve exhibits little enhancement (solid arrow), in contrast to the DTF, which shows avid enhancement (asterisk). Subtle signal increase on an axial proton density scan with fat suppression (C) can be seen in the superior gluteal nerve (solid arrow) as it passes through the sciatic notch toward the gluteus medius and minimus.

$>50 \%$ intralesional fat. In addition, the spectrum of clinical findings with NMC manifests nerve-territory undergrowth including muscular atrophy, bone dysplasia, and limb length discrepancy, ${ }^{14}$ whereas LFH is associated with nerve-territory bone and soft-tissue overgrowth. ${ }^{19,28}$ The natural history of NMC and NMC-associated DTF is still not known. Given the strong association between biopsy or excision of NMC and the subsequent development of DTF, we advocate a "no-touch" technique-i.e., the diagnosis should be based solely on clinical and imaging criteria. ${ }^{10}$ Patients are typically diagnosed during adolescence or young adulthood; a high index of suspicion is required, and physical examination can be supplemented by quantitative measurement techniques for limb length discrepancy such as scanography.

The association of NMC with an aggressive fibromatosis has been described only recently. Given the rarity of NMC, particularly in contrast to sporadic DTF, the diagnosis of NMC-associated fibromatosis may reasonably be overlooked. We speculate that a recently published case of myofibromatosis presenting with developmental dysplasia of the hip may actually represent NMC-associated fibromatosis (instead of myofibromatosis) with unrecognized NMC of the lumbosacral plexus and sciatic nerve ("sciatic nerve was inseparable from the tumour") and associated bony undergrowth leading to dysplasia. ${ }^{33}$ Nerve abnormalities suggestive of NMC are well seen distal to the mass on sagittal image Fig. 3C in that case report. Based on the description (no histological images were included), the lesion was composed of both cytologically bland (myo)fibroblasts forming long fascicles and a smooth muscle component. The description of the myofibroblastic component forming long fascicles would support the diagnosis of a DTF, regardless of the beta-catenin immunostain, which not infrequently shows an equivocal pattern of nuclear and cytoplasmic staining in DTF; admittedly, however, the presence of smooth muscle would be unusual in DTF. A further literature search revealed two case reports of desmoplastic fi- broma (recurrent desmoid tumors) that exhibited "perineural spread" after resection and aggressive recurrence. . $^{20} 2$ Both cases were closely associated with enlarged nerves, leading the authors to conclude that the DTF invaded local nerve tissue. It is our opinion, however, that these reports may actually represent other examples of occult NMC associated with DTF that have recurrent aggressive behavior.

We have recently demonstrated that NMC and NMC-associated DTF both harbor identical mutations in CTNNB1, particularly $C T N N B 1$ p.S45F, a specific CTNNB1 mutation that has been associated with a more aggressive clinical behavior. ${ }^{2}$ While the $C T N N B 1$ mutation is not specific to $\mathrm{NMC}$ or NMC-associated fibromatosis, in our experience, it is present in $>90 \%$ of cases, whereas CTNNBI p.S45F mutations are much less common than other CTNNB1 mutations in sporadic DTF. CTNNB1 encodes beta-catenin and activating exon 3 mutations in CTNNB1, which are present in $85 \%-90 \%$ of sporadic DTFs, resulting in aberrant beta-catenin/Wnt signaling. In patients with NMC, we suspect that a pluripotent ectomesenchymal precursor harbors the CTNNB1 mutation, manifesting as an NMC and also "priming" the surrounding (myo) fibroblasts for the development of DTF. This may account for the strong association of NMC with the development of NMC-associated DTF following iatrogenic injury (i.e., biopsy). Peripheral nerves have an established role in normal tissue healing, ${ }^{16}$ and beta-catenin expression is upregulated following traumatic nerve injury. ${ }^{15}$ The presence of a CTNNB1 mutation throughout an NMC-affected nerve and surrounding soft tissues may also account for the presence of additional foci of DTF found in our cases 1 and 3.

\section{Treatment Implications}

While sporadic DTF accounts for 3\% of all soft-tissue tumors, ${ }^{8}$ there is likely a subset that is associated with occult NMC. The three patients presented in this report may serve as initial experience with NMC-associated desmoid 

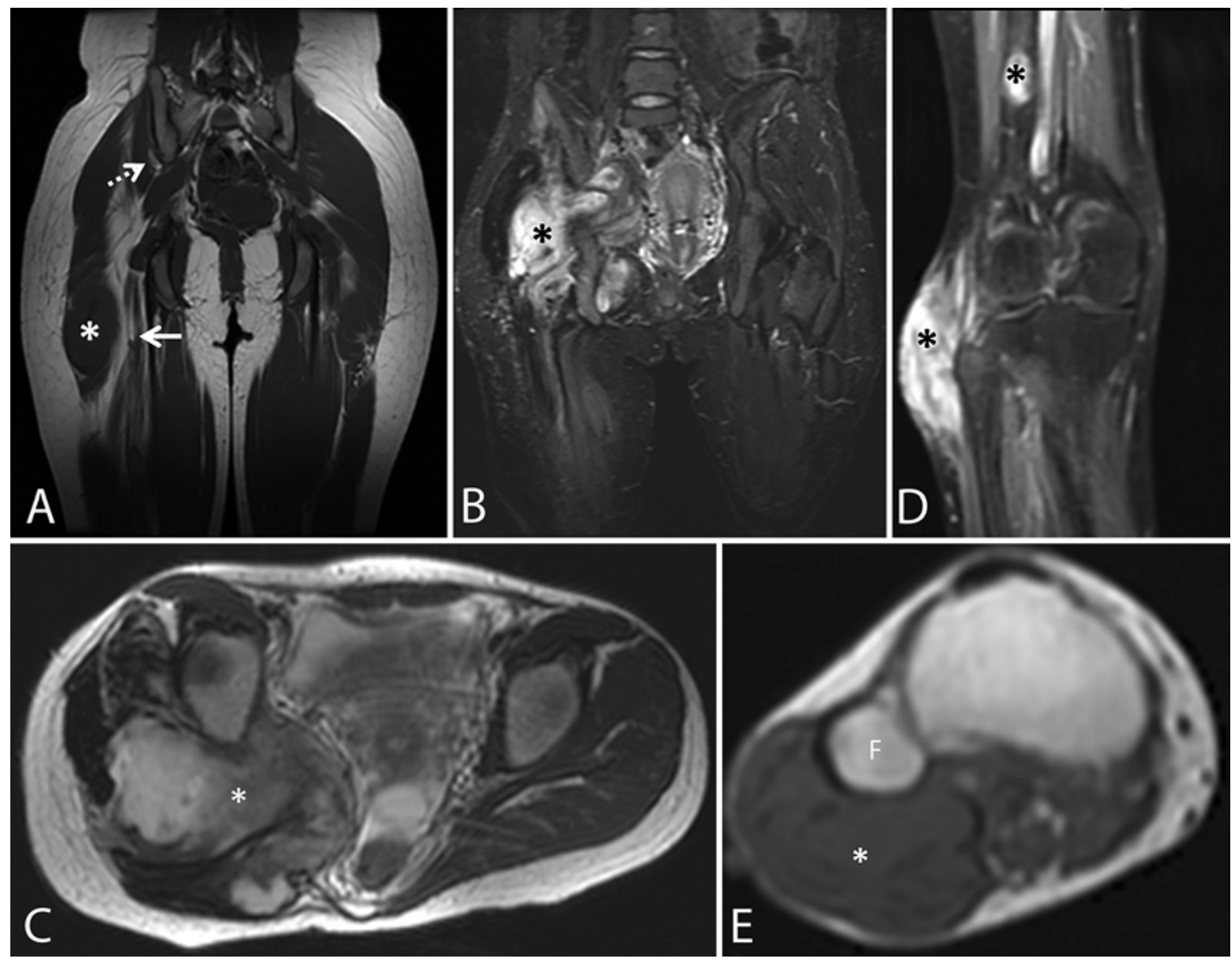

FIG. 7. Case 3. Preoperative coronal T1-weighted image (A) showing the DTF (asterisk) in the gluteus maximus adjacent to an enlarged sciatic nerve (solid arrow) and superior gluteal nerve (dotted arrow). Postoperative MRI (B-E) was performed 26 months after resection of the proximal sciatic DTF. Coronal short-tau inversion recovery (STIR) image (B) showing recurrence of the DTF (asterisk) along the course of the abnormal muscular branch, extending into the pelvis via the greater sciatic notch. Axial T2 fast spin echo (FSE) sequence (C) without fat suppression obtained at the level of the sciatic notch, showing a large heterogeneous mass extending along the sciatic nerve into the pelvis (asterisk). Coronal T1-weighted image with fat suppression after Gd contrast (D) showing two distal foci of DTF in the popliteal fossa (asterisks). Axial T1-weighted image (E) showing the DTF (asterisk) wrapping around the fibular head (F), along the course of the lateral sural cutaneous nerve (not visualized due to size of tumor).

tumors; however, this entity is likely underdiagnosed and its true incidence is not known. Given the aggressive nature of NMC-associated DTF, there needs to be a high index of suspicion for NMC when initially evaluating patients with sporadic DTF. We believe that the finding of DTF in proximity to nerves is not coincidental and that nerves may have a role in their localization, progression, and recurrence. Peripheral nerves should be scrutinized carefully on MRI in all patients with newly discovered DTF of the extremities. Furthermore, if there are any abnormal nerve findings suggestive of NMC, MRI of the entire extremity should be considered, as we suspect this pathology may predispose to multifocal disease.

In this small patient series, the NMC-associated DTF seemed to behave aggressively with significant recurrence and the development of multifocal DTF along the course of the affected nerve. For sporadic DTF, the Italian and French Sarcoma Group endorsed a period of watchful waiting and a trial of anti-hormonal agents. ${ }^{6,9}$ With tumor progression, aggressive resection is recommended if feasible. For patients with NMC-associated DTF, the correct treatment algorithm is unknown. This subgroup of patients poses a difficult scenario because surgery itself is known to incite DTF formation ${ }^{10}$ and the multifocal pattern of DTF formation may lead to further neoplastic lesions at other sites in the same extremity. Even with amputation, it is unlikely that further DTF formation will be prevented: for example, the NMC can appear more proximally, and DTF formation can occur well above the level of the amputation (as seen with case 3). We anticipate that the mainstay of treatment for NMC-associated DTF will be nonsurgical management (except for biopsy) including loco-regional chemotherapy (e.g., isolated limb perfusion with tumor necrosis factor alpha and melphalan), exclusive radiation, systemic chemotherapy (doxorubicin or Vinca alkaloids), and/or targeted therapy (e.g., imatinib or sorafenib). ${ }^{9}$

An important aspect of counseling women diagnosed with DTF is the $50 \%$ risk of DTF progression during pregnancy? This may be especially important for patients with occult NMC. The patient in case 3 developed an aggressive recurrence and a new lesion distal to her primary tumor during pregnancy. While the true risk is unknown, 
it may be prudent to advise against pregnancy for women who harbor NMC for fear that an NMC-associated DTF may occur.

\section{Study Limitations}

We acknowledge the limitations of any retrospective review. Given the referral nature of care, there was limited information regarding clinical examination, diagnostic tests, disease course, treatments, and follow-up for the patients. Similarly, it is impossible to know the true extent of resection of DTF based on the operative notes from other institutions, and unfortunately immediate postoperative MRI was not performed. While we suspect that NMC-associated DTF exhibits an aggressive phenotype, it is possible that initial subtotal resection led to local recurrence.

The available MRI studies for many of the reviewed patients in our series were low resolution and did not capture the full course of the pathological nerves in the cases. It is possible that the rate of NMC was actually higher and that some cases were missed in this review. Similarly, other published reports of DTFs adjacent to nerve typically do not include sufficient MR images to evaluate for the presence of an occult NMC.

Pathological confirmation of the NMC was only available in case 1. Even without this information for cases 2 and 3, we are confident about the presence of NMC because the radiological findings were pathognomonic. While we are cautious in drawing conclusions from retrospectively collected data, NMC is an extremely rare lesion, and its strong association with DTF, particularly following biopsy or resection, is unlikely to be coincidental.

\section{Conclusions}

Through these three cases, we provide a rationale for maintaining a high index of suspicion for NMC when assessing patients with seemingly sporadic DTF. Peripheral nerves should be scrutinized carefully for evidence of NMC on MRI in all patients with newly discovered DTF of the extremities because of the potential diagnostic and treatment implications. Interestingly, NMC-associated DTF may represent a subtype of the disease with unique biological behavior.

\section{References}

1. Antonescu CR, Scheithauer BW, Woodruff JM (eds): Tumors of the Peripheral Nervous System. Bethesda, MD: American Registry of Pathology, 2013

2. Carter JM, Howe BM, Hawse JR, Giannini C, Spinner RJ, Fritchie KJ: CTNNB1 mutations and estrogen receptor expression in neuromuscular choristoma and its associated fibromatosis. Am J Surg Pathol 40:1368-1374, 2016

3. Dafford K, Kim D, Nelson A, Kline D: Extraabdominal desmoid tumors. Neurosurg Focus 22(6):E21, 2007

4. Dafford K, Kim D, Reid N, Kline D: Pelvic plexus tumors. Neurosurg Focus 22(6):E10, 2007

5. Ferraresi S, Garozzo D, Bianchini E: Aggressive fibromatosis (desmoid tumor) of the radial nerve: favorable resolution. Case report. J Neurosurg 95:332-333, 2001

6. Fiore M, Colombo C, Radaelli S, Callegaro D, Palassini E, Barisella M, et al: Hormonal manipulation with toremifene in sporadic desmoid-type fibromatosis. Eur J Cancer 51:28002807, 2015

7. Fiore M, Coppola S, Cannell AJ, Colombo C, Bertagnolli MM, George S, et al: Desmoid-type fibromatosis and pregnancy: a multi-institutional analysis of recurrence and obstetric risk. Ann Surg 259:973-978, 2014

8. Fletcher CDM, Bridge JA, Hogendoorn P, Mertens F (eds): WHO Classification of Tumours, in WHO Classification of Tumours of Soft Tissue and Bone. Pathology and Genetics of Tumours of Soft Tissue and Bone, ed 4. Lyon: IARC Press, 2013, Vol 5

9. Gronchi A, Colombo C, Le Péchoux C, Dei Tos AP, Le Cesne A, Marrari A, et al: Sporadic desmoid-type fibromatosis: a stepwise approach to a non-metastasising neoplasm-a position paper from the Italian and the French Sarcoma Group. Ann Oncol 25:578-583, 2014

10. Hébert-Blouin MN, Amrami KK, Spinner RJ: Addendum: Evidence supports a "no-touch" approach to neuromuscular choristoma. J Neurosurg 119:252-254, 2013

11. Hébert-Blouin MN, Scheithauer BW, Amrami KK, Durham SR, Spinner RJ: Fibromatosis: a potential sequela of neuromuscular choristoma. J Neurosurg 116:399-408, 2012

12. Huang JH, Samadani U, Zager EL: Case studies for illustration and discussion: peripheral nerve tumors. Neurosurg Clin N Am 15:241-249, viii, 2004

13. Joglekar SB, Rose PS, Sim F, Okuno S, Petersen I: Current perspectives on desmoid tumors: the Mayo Clinic approach. Cancers (Basel) 3:3143-3155, 2011

14. Kumar R, Howe BM, Amrami KK, Spinner RJ: Neuromuscular choristoma of the sciatic nerve and lumbosacral plexus: an association with nerve-territory undergrowth in the pelvis affecting soft tissue and bone. Acta Neurochir (Wien) 156:1041-1046, 2014

15. Kurimoto S, Jung J, Tapadia M, Lengfeld J, Agalliu D, Waterman M, et al: Activation of the Wnt//-catenin signaling cascade after traumatic nerve injury. Neuroscience 294:101108,2015

16. Laverdet B, Danigo A, Girard D, Magy L, Demiot C, Desmoulière A: Skin innervation: important roles during normal and pathological cutaneous repair. Histol Histopathol 30:875-892, 2015

17. Lazar AJF, Tuvin D, Hajibashi S, Habeeb S, Bolshakov $\mathrm{S}$, Mayordomo-Aranda E, et al: Specific mutations in the $\beta$-catenin gene (CTNNB1) correlate with local recurrence in sporadic desmoid tumors. Am J Pathol 173:1518-1527, 2008

18. Lee CH, Wu JS, Goldsmith JD, Kung JW: Lipomatosis of the sciatic nerve secondary to compression by a desmoid tumor. Skeletal Radiol 42:1751-1754, 2013

19. Mahan MA, Niederhauser BD, Amrami KK, Spinner RJ: Long-term progression of lipomatosis of nerve. World Neurosurg 82:492-499, 2014

20. Moorjani V, Stockton V: Desmoplastic fibroma with perineural extension. AJR Am J Roentgenol 185:1498-1499, 2005

21. Niederhauser BD, Spinner RJ, Howe BM, Amrami KK: In response to "Lipomatosis of the sciatic nerve secondary to compression by a desmoid tumor." Skeletal Radiol 43:129, 2014

22. Niederhauser BD, Spinner RJ, Jentoft ME, Everist BM, Matsumoto JM, Amrami KK: Neuromuscular choristoma: characteristic magnetic resonance imaging findings and association with post-biopsy fibromatosis. Skeletal Radiol 42:567-577, 2013

23. Özger H, Özkunt O, Akgül T, Sağlam Y: Preventing neurovascular invasion in desmoid tumors. Acta Orthop Traumatol Turc 47:286-290, 2013

24. Polveche G, Langeron P, Saout J: [Extra-abdominal desmoid tumors. Apropos of a case in the calf.] J Chir (Paris) 123:255-260, 1986 (Fr)

25. Press JM, Rayner SL, Philip M, Monga TN, Katz RT: 
Intraoperative monitoring of an unusual brachial plexus tumor. Arch Phys Med Rehabil 73:297-299, 1992

26. Ribeiro BN, Salata TM, Antunes Lde O, Marchiori E: Desmoplastic fibroma with perineural spread: conventional and diffusion-weighted magnetic resonance imaging findings. Radiol Bras 48:266-267, 2015

27. Seinfeld J, Kleinschmidt-Demasters BK, Tayal S, Lillehei KO: Desmoid-type fibromatoses involving the brachial plexus: treatment options and assessment of c-KIT mutational status. J Neurosurg 104:749-756, 2006

28. Silverman TA, Enzinger FM: Fibrolipomatous hamartoma of nerve. A clinicopathologic analysis of 26 cases. Am J Surg Pathol 9:7-14, 1985

29. Siqueira MG, Tavares PL, Martins RS, Heise CO, Foroni LHL, Bordalo M, et al: Management of desmoidtype fibromatosis involving peripheral nerves. Arq Neuropsiquiatr 70:514-519, 2012

30. Soldatos T, Andreisek G, Thawait GK, Guggenberger R, Williams EH, Carrino JA, et al: High-resolution 3-T MR neurography of the lumbosacral plexus. Radiographics 33:967-987, 2013

31. Wadhwa V, Thakkar RS, Maragakis N, Höke A, Sumner CJ, Lloyd TE, et al: Sciatic nerve tumor and tumor-like lesionsuncommon pathologies. Skeletal Radiol 41:763-774, 2012

32. Weschenfelder W, Lindner R, Spiegel C, Hofmann GO, Vogt M: Desmoid tumor of the popliteal fossa during pregnancy.

Case Rep Surg 2015:262654, 2015
33. Woo T, Win T: An unusual cause of neonatal hip dislocation: infantile myofibromatosis presenting as developmental dysplasia of the hip. Hip Int 26:e39-e41, 2016

\section{Disclosures}

The authors report no conflict of interest concerning the materials or methods used in this study or the findings specified in this paper.

\section{Author Contributions}

Conception and design: Spinner, Howe, Amrami. Acquisition of data: Spinner, Stone, Prasad, Laumonerie, Howe, Amrami, Jentoft. Analysis and interpretation of data: all authors. Drafting the article: Spinner, Stone, Prasad, Laumonerie, Howe, Carter, Jentoft. Critically revising the article: all authors. Reviewed submitted version of manuscript: Spinner, Stone, Prasad, Laumonerie, Carter, Jentoft. Approved the final version of the manuscript on behalf of all authors: Spinner. Study supervision: Spinner.

\section{Correspondence}

Robert J. Spinner: Mayo Clinic, Rochester, MN. spinner.robert@ mayo.edu. 